Enferm Bras 2020;19(3):202-10

https://doi.org/10.33233/eb.v19i3.2351

\title{
ARTIGO ORIGINAL \\ Estudo retrospectivo de radiodermatite em pacientes com câncer de mama: uma experiência institucional privada e pública
}

Andrea Moraes Ipiranga*, Cintia Yolette Urbano Pauxis Aben-Athar, D.Sc.**, Renata Glaucia Barros da Silva Lopes, M.Sc. ${ }^{* \star *}$, Heryvelton Lima de Freitas ${ }^{\star * *}$, Aline Maria Pereira Cruz Ramos, D.Sc. ${ }^{* * * *}$

*Enfermeira, Especialista em Enfermagem Oncológica, Hospital Porto Dias, Belém/PA, ${ }^{* *}$ Enfermeira, Faculdade de Enfermagem, Universidade Federal do Pará, Belém/PA, ${ }^{* * \star}$ Enfermeira, Universidade da Amazônia, Belém/PA, ${ }^{* * * * M e ́ d i c o ~ e s p e c i a l i s t a ~ e m ~ r a d i o t e r a p i a ~}$ do Hospital Porto Dias e Hospital Ophir Loyola, Belém/PA, ${ }^{\star * * * \star E n f e r m e i r a, ~ F a c u l d a d e ~ d e ~}$ Enfermagem/UFPA e Núcleo de Pesquisa em Oncologia/UFPA, Belém/PA

Recebido em 18 de maio de 2019; aceito em 22 de maio de 2020.

Correspondência: Aline Maria Pereira Cruz Ramos, Faculdade de Enfermagem da UFPA e Núcleo de Pesquisa em Oncologia em Oncologia, Tv. Benjamim Constant, 631, 66053-040 Belém PA

Andrea Moraes Ipiranga: dedeia_ipiranga@hotmail.com

Cintia Yolette Urbano Pauxis Aben-Athar: abenathar_cintia@hotmail.com

Renata Glaucia Barros da Silva Lopes: renatagbsilva@yahoo.com.br

Heryvelton Lima de Freitas: heryvelton@me.com

Aline Maria Pereira Cruz Ramos: nurse.alinecruz@gmail.com

\section{Resumo}

Introdução: A radiodermatite aguda é comumente observada em pacientes com câncer de mama submetidas à radioterapia. Objetivo: Comparar a radiodermatite aguda em mulheres tratadas com radioterapia convencional ou com intensidade modulada em dois Centros de Radioterapia (intituição pública e privada). Métodos: Estudo transversal, retrospectivo e descritivo, desenvolvido em dois hospitais da cidade de Belém/PA, que avaliou 343 mulheres entre 2012 e 2015. Resultados: Houve radiodermatite aguda nos dois Centros de Radioterapia analisados, coincidindo entre a $16^{\circ}$ à $20^{\circ}$ aplicação independentemente do tipo de tecnologia utilizada. A maioria das lesões foram classificadas como RTOG1 e interrupção do tratamento por 1 semana ocorreu na instituição pública. Quanto ao manejo profilático, as instituições divergiram na indicação da compressão com chá de camomila e creme a base de aloé vera, e coincidiram no tratamento farmacológico com esteróide e antibiótico tópico. Conclusão: Os resultados evidenciaram radiodermatite aguda em ambas instituições, independente da técnica aplicada.

Palavras-chave: neoplasia de mama, radioterapia, radiodermatite.

Abstract

Retrospective study of radiodermatitis in breast cancer patients: a public and private institutional experience

Introduction: Acute radiodermatitis is commonly seen in patients with breast cancer undergoing radiation therapy. Aim: To compare acute radiodermatitis in women treated with conventional radiotherapy or modulated intensity in two Radiotherapy Centers (public and private institutions). Methods: Cross-sectional, retrospective and descriptive study, developed in two hospitals in the city of Belém (in the north of Brazil), which evaluated 343 women between 2012 and 2015. Results: There was acute radiodermatitis in the two Radiotherapy Centers analyzed, coinciding between 16th to 20th application regardless of the type of technology used. Most injuries were classified as RTOG1, and treatment interruption for 1 week occurred in the public institution. As for prophylactic management, the institutions diverged in the indication of compression with chamomile tea and cream based on aloe vera and coincided in the pharmacological treatment 
with steroid and topical antibiotic. Conclusion: The results showed acute radiodermatitis in both institutions, regardless of the technique applied.

Keywords: breast neoplasms, radiotherapy, radiodermatitis.

\section{Resumen}

Estudio retrospectivo de radiodermatitis en pacientes con cáncer de mama: una experiencia institucional privada y publica

Introducción: La radiodermatitis aguda se observa comúnmente en pacientes con cáncer de mama que reciben radioterapia. Objetivo: Comparar la dermatitis aguda en mujeres tratadas con radioterapia convencional o con intensidad modulada en dos centros de radioterapia (instituciones públicas y privadas). Métodos: Estudio transversal, retrospectivo y descriptivo, desarrollado en dos hospitales de la ciudad de Belém (en el norte de Brasil), que evaluó a 343 mujeres entre 2012 y 2015. Resultados: Radiodermatitis aguda en los dos Centros de Radioterapia analizados, coincidiendo entre 16 y 20 fracciones, independientemente del tipo de tecnología utilizada. La mayoría de las lesiones se clasificaron como RTOG1 y la interrupción del tratamiento durante 1 semana ocurrió en la institución pública. En cuanto al tratamiento profiláctico, las instituciones divergieron en la indicación de compresión con té de manzanilla y crema a base de aloe vera, y coincidieron en el tratamiento farmacológico con esteroides y antibióticos tópicos. Conclusión: Los resultados mostraron radiodermatitis aguda en ambas instituciones, independientemente de la técnica aplicada.

Palabras-clave: neoplasias de la mama, radioterapia, radiodermatitis.

Introdução

O câncer de mama é o tipo mais comum de câncer em mulheres [1] e a radioterapia é um componente crítico e comum no tratamento desta doença [2].

Apesar dos avanços tecnológicos e da melhor dosimetria loco-regional alcançados pela radioterapia de intensidade modulada (IMRT), esta ainda é capaz de causar toxicidade cutânea devido à pele estar entre a fonte de radiação e o alvo terapêutico, caracterizando-a como fator dose-limitante no controle da doença devido à interrupção prematura, dose e prognóstico [3]. Mais de $90 \%$ das mulheres em radioterapia experimentam algum grau de radiodermatite no decorrer do tratamento ou imediatamente após, afetando a autoimagem e qualidade de vida [4,5].

A radiodermite é complexa e multifatorial estando relacionada a fatores intrínsecos e extrínsecos (principalmente, relacionados a dose total, diária, boost, tipo de energia, etc.) [6,7]. Esta lesão aguda surge entre 10-14 dias após o início do tratamento e a acumulação de dose induz à interrupção da mitose, regeneração celular e apoptose, bem como a indução de processo inflamatório no tecido e danos endoteliais [8]. Assim, o enfermeiro é capaz de atrasar o surgimento e diminuir a gravidade da radiodermatite desde que as orientações de autocuidado sejam seguidas e haja adesão ao tratamento, como a aplicação de produtos profiláticos [9].

Este estudo teve como objetivo comparar a radiodermatite aguda em mulheres tratadas com radioterapia convencional (3D-CRT) ou com intensidade modulada em dois Centros de Radioterapia (instituição pública e privada).

Material e métodos

Trata-se de um estudo transversal, retrospectivo e descritivo, desenvolvido em dois hospitais da cidade de Belém (no norte do Brasil), o Hospital Ophir Loyola e Hospital Porto Dias (HPD), instituições públicas e privadas, respectivamente, no período entre 2012 e 2015. Este estudo foi submetido ao Comitê de Ética em Pesquisa sob pareceres № 904.233 e 1.398.679.

A população foi composta por 343 mulheres com histopatológico positivo para câncer de mama tratadas com radioterapia durante o mesmo período nas duas instituições. Foram excluídos pacientes com câncer de mama masculino e mulheres sem indicação de radioterapia.

Foi aplicado um formulário criado pelos autores para direcionar e otimizar a coleta de dados dos prontuários, cujas variáveis identificadas estavam relacionadas a dados epidemiológicos, clínicos-patológicos e terapêuticos. A análise dos dados ocorreu em duas fases, a primeira foi descritiva à incidência de radiodermatite aguda nas diferentes tecnologias (IMRT vs. 3D-CRT) e a segunda comparou o grau de RTOG às variáveis relacionadas ao tratamento de cada tipo de tecnologia aplicada. 
Os dados foram organizados em planilhas no Programa Excel 2010 e submetidos à análise estatística descritiva contendo a frequência e a porcentagem de todas as variáveis relacionadas aos achados clínicos e às características epidemiológicas dos participantes. $\mathrm{Na}$ análise inferencial, o teste exato de Fisher e o qui-quadrado $\left(\chi^{2}\right)$ foram utilizados nas análises de variáveis categóricas em relação às reações cutâneas agudas (RTOG) e o teste G foi utilizado na comparação da radiodermatite aguda entre instituições públicas e privadas. Todas as análises estatísticas foram realizadas usando o Pacote $\mathrm{R}$ v2.15 e foram fixadas em um nível significativo de $5 \%$.

\section{Resultados}

A análise dos prontuários de 343 mulheres mostrou uma idade média de 55 anos $( \pm 24$ $95)$, sendo provenientes da região metropolitana de Belém (65,3\%). A maioria delas apresentou carcinoma ductal invasivo $(74,3 \%)$, acometendo a mama direita $(39,6 \%)$ ou $(34,1 \%)$ a esquerda, com subtipo Luminal A ou B por Imuno-histoquímica entre os dois grupos (Tabela I). Interessantemente, o estadiamento tumoral divergiu entre as instituições, prevaleceu o precoce (TNM I + II com 36,1\%) na privada e tardio (TNM III com 25\%) na pública (Figura 1).

Tabela I - Características patológicas clínicas dos dois grupos de pacientes.

\begin{tabular}{|c|c|c|}
\hline Variável & Instituição pública (3D-CRT) & $\begin{array}{l}\text { Instituição privada (IMRT) } \\
\text { N \% }\end{array}$ \\
\hline Idade (anos), média & $55(24-95)$ & \\
\hline \multicolumn{3}{|l|}{ Origem (\%) } \\
\hline $\begin{array}{l}\text { Região metropolitana } \\
\text { Interior } \\
\text { Outros / sem registro }\end{array}$ & $\begin{array}{l}100(49,01) \\
53(25,98) \\
51(25,01)\end{array}$ & $\begin{array}{l}124(89,20) \\
5(3,60) \\
10(7,19)\end{array}$ \\
\hline \multicolumn{3}{|l|}{ Lado } \\
\hline $\begin{array}{l}\text { Direito } \\
\text { Esquerdo } \\
\text { Sem registro } \\
\end{array}$ & $\begin{array}{l}59(28,92) \\
54(26,48) \\
91(44,60) \\
\end{array}$ & $\begin{array}{l}77(55,39) \\
63(44,61) \\
0(0)\end{array}$ \\
\hline \multicolumn{3}{|l|}{ Histologia } \\
\hline $\begin{array}{l}\text { Carcinoma ductal invasivo } \\
\text { Carcinoma lobular invasivo } \\
\text { Outros / sem registro }\end{array}$ & $\begin{array}{l}146(71,56) \\
6(2,95) \\
52(25,49)\end{array}$ & $\begin{array}{l}109(78,41) \\
7(5,05) \\
23(16,54)\end{array}$ \\
\hline \multicolumn{3}{|l|}{ Immunohistoquímica } \\
\hline Luminal A & $16(7,9)$ & $29(20,8)$ \\
\hline Luminal B & $7(3,5)$ & $39(28,3)$ \\
\hline Superexpressão Her 2 & $1(0,5)$ & $13(9,3)$ \\
\hline Triplo Negativo & $5(2,4)$ & $15(10,7)$ \\
\hline Sem registro & $175(85,7)$ & $43(30,9)$ \\
\hline \multicolumn{3}{|l|}{ TNM *a } \\
\hline 1 & $3(0,9)$ & $56(33)$ \\
\hline ॥ & $31(15,3)$ & $34(24,8)$ \\
\hline III & $51(25)$ & $29(20,8)$ \\
\hline IV & $0(0)$ & $0(0)$ \\
\hline Sem registro & $119(58,3)$ & $20(14,3)$ \\
\hline \multicolumn{3}{|l|}{ Cirurgia } \\
\hline $\begin{array}{l}\text { Conservadora } \\
\text { Radical modificada } \\
\text { Nenhum/sem registro }\end{array}$ & $\begin{array}{l}100(49,01) \\
100(49,01) \\
4(1,98)\end{array}$ & $\begin{array}{l}91(65,46) \\
46(33,09) \\
2(1,45)\end{array}$ \\
\hline
\end{tabular}




\begin{tabular}{lll}
\hline \multicolumn{2}{l}{ Quimioterapia concomitante } & \\
\hline Sim & $18(8,83)$ & $91(65,46)$ \\
Não & $177(86,76)$ & $46(33,09)$ \\
Outros / sem registro & $9(4,41)$ & $2(1,45)$ \\
\hline Esquema de quimioterapia*b & & \\
\hline AC & $32(15,68)$ & $3(2,18)$ \\
AC-T & $121(59,31)$ & $48(34,53)$ \\
CMF & $12(5,88)$ & $4(2,87)$ \\
FAC & $7(3,45)$ & $4(2,87)$ \\
Nenhum / sem registro & $32(15,68)$ & $80(57,55)$ \\
\hline
\end{tabular}

${ }^{* a}$ Classificação TNM de tumores malignos, $8^{\circ}$ ed [18]; *b Diretrizes Diagnósticas e Terapêuticas do Carcinoma de Mama (Portaria no 04/2018) [19].

Identificou-se também divergência entre as instituições quanto a cirurgia e quimioterapia, o que foge do escopo desta análise. Ressalta-se a grande quantidade de informações ausentes e/ou incompletas, com destaque aos registos da imunohistoquímica e estadiamento nas mulheres da instituição pública.

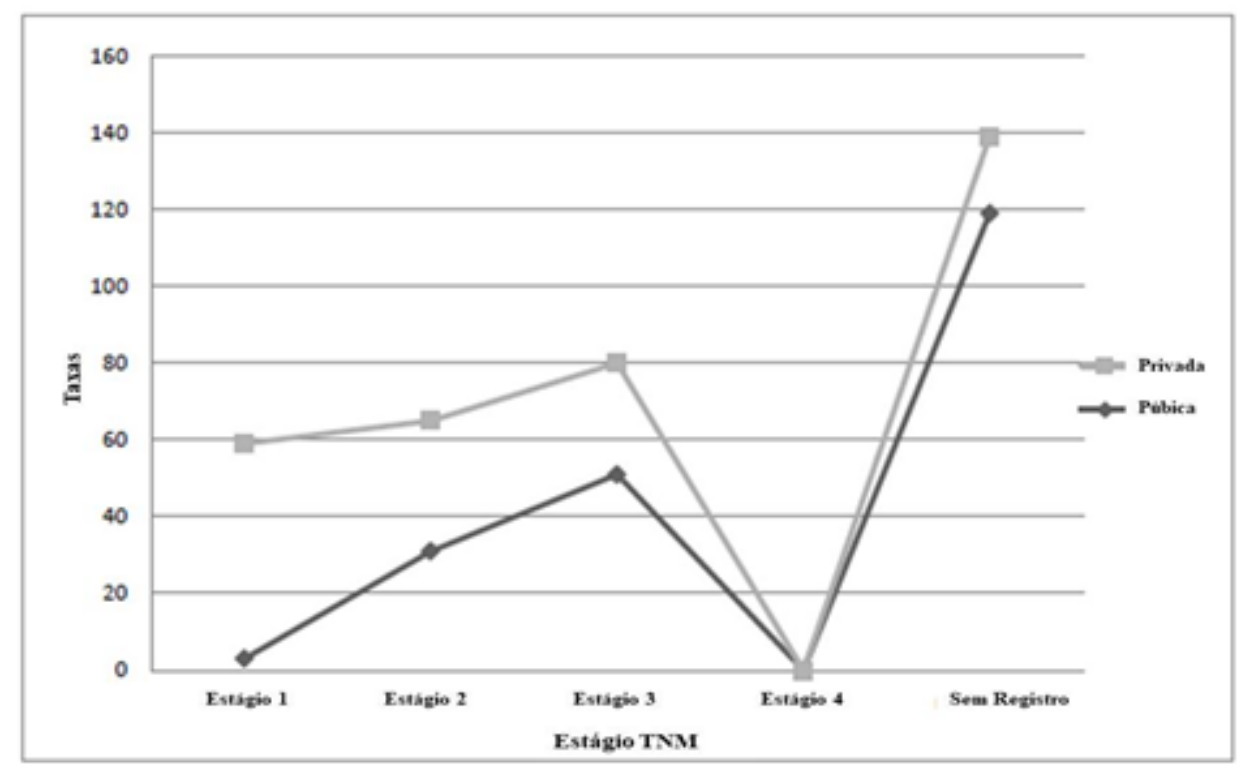

Figura 1 - Níveis de estadiamento de câncer de mama em pacientes do gênero feminino de um Hospital Público e Privado. Belém/PA, Brasil, 2012-2015.

A figura 2 mostra o pico de radiodermatite aguda entre o $16^{\circ}$ e $20^{\circ}$ dia de aplicação de radioterapia, independentemente do tipo de tecnologia utilizada (instituição pública $24 \%$ e privada $28,77 \%)$. A maioria delas foi classificada como RTOG1, com destaque na instituição privada $(74,1 \%)$. Alternativamente, as mulheres da instituição pública apresentaram lesões de grau $2(20,58 \%)$ e $3(5,41 \%)$ (Tabela II). Nenhuma toxicidade aguda de Grau 4 ou superior foi observada em ambas as instituições (Figura 3).

A tabela II mostrou variação significativa quanto ao protocolo terapêutico seguido nas instituições. A tecnologia IMRT aplicou maior dose total $(p=0,0001)$, maior fração diária $(p=0,0001)$ e menor número de sessões $(p=0,0001)$, caracterizando-se tratamento hipofracionado. Por outro lado, a instituição pública, com tecnologia 3D-CRT, ofertou menor dose total, equiparação de dose diária e maior número de sessões, caracterizando-se como tratamento hiperfracionado. Na minoria das pacientes o boost foi aplicado, com destaque para a instituição pública $(p=0,0397)$. 
Tabela II - Análise de RTOG em comparação aos fatores relacionados à radioterapia por instituição.

\begin{tabular}{|c|c|c|c|c|c|}
\hline \multirow[t]{2}{*}{ Dose total } & \multicolumn{2}{|c|}{$\begin{array}{l}\text { Instituição pública } \\
\text { (3D-CRT) }\end{array}$} & \multicolumn{2}{|c|}{$\begin{array}{l}\text { Instituição privada } \\
\text { (IMRT) }\end{array}$} & \multirow{2}{*}{$\begin{array}{l}p \text { valor } \\
\text { 3D-CRT x } \\
\text { IMRT }\end{array}$} \\
\hline & $\%$ & $\begin{array}{l}\text { RTOG } \\
\text { ( } p \text { valor) }\end{array}$ & $\%$ & $\begin{array}{l}\text { RTOG } \\
\text { ( } p \text { valor) }\end{array}$ & \\
\hline \multicolumn{6}{|l|}{$\begin{array}{l}\text { Dose total de radiação } \\
\text { ionizante }\end{array}$} \\
\hline $\begin{array}{l}30-45 \text { Gys } \\
>45 \text { Gys } \\
\text { Sem registro }\end{array}$ & $\begin{array}{l}132(64,70) \\
69(33,82) \\
3(1,48)\end{array}$ & - & $\begin{array}{l}25(17,98) \\
112(80,57) \\
2(1,45)\end{array}$ & $0,00024^{*}$ & 0,0001 \\
\hline \multicolumn{6}{|l|}{ Dose por fração } \\
\hline $\begin{array}{l}1,8 \text { Gy } \\
\geq 2 \text { Gys } \\
\text { Sem registro }\end{array}$ & $\begin{array}{l}91(44,60) \\
110(53,92) \\
3(1,48)\end{array}$ & - & $\begin{array}{l}9(6,47) \\
128(92,08) \\
2(1,45)\end{array}$ & - & $0,0001^{* * *}$ \\
\hline \multicolumn{6}{|l|}{ Número de sessões } \\
\hline $\begin{array}{l}<20 \\
20-25 \\
26-30 \\
>31 \\
\text { Sem registro }\end{array}$ & $\begin{array}{l}0(0) \\
57(27,94) \\
90(44,11) \\
32(15,68) \\
25(12,27)\end{array}$ & - & $\begin{array}{l}21(15,10) \\
103(74,10) \\
13(9,35) \\
0(0) \\
2(1,45)\end{array}$ & - & $0,0001^{* * *}$ \\
\hline \multicolumn{6}{|l|}{ Boost } \\
\hline $\begin{array}{l}\text { Sim } \\
\text { Não } \\
\text { Nenhum/sem registro }\end{array}$ & $\begin{array}{l}67(32,84) \\
134(65,68) \\
3(1,48)\end{array}$ & - & $\begin{array}{l}29(20,86) \\
110(79,13) \\
1(0,01)\end{array}$ & - & $0,0397^{* * *}$ \\
\hline
\end{tabular}

\section{Diagnóstico de radiodermatite (№ sessão)}

\begin{tabular}{|c|c|c|c|c|}
\hline $9-15$ & $26(12,74)$ & $\mathrm{p}=0,003785$ & $26(18,70)$ & $2,2 \times 10^{-6 * *} 0,0004^{* *}$ \\
\hline $16-20$ & $49(24,01)$ & & $40(28,77)$ & \\
\hline $21-25$ & $37(18,13)$ & & $36(25,89)$ & \\
\hline$>25$ & $16(7,87)$ & & $16(11,54)$ & \\
\hline Nenhum/sem registro & $76(37,25)$ & & $21(15,10)$ & \\
\hline
\end{tabular}

\section{Grau de RTOG durante tratamento}

\begin{tabular}{|c|c|c|c|c|c|}
\hline $\begin{array}{l}\text { I } \\
\text { II } \\
\text { III } \\
\text { IV } \\
\text { Nenhum/sem registro }\end{array}$ & $\begin{array}{l}75(36,76) \\
42(20,58) \\
11(5,41) \\
0(0) \\
76(37,25)\end{array}$ & - & $\begin{array}{l}103(74,1) \\
19(13,66) \\
1(0,7) \\
0(0) \\
16(11,54)\end{array}$ & - & $0,0001^{* * *}$ \\
\hline \multicolumn{6}{|l|}{ Interrupção no tratamento } \\
\hline $\begin{array}{l}1 \text { Semana } \\
2 \text { Semanas } \\
>2 \text { Semanas } \\
\text { Nenhum/Sem registro }\end{array}$ & $\begin{array}{l}10(4,90) \\
9(4,41) \\
4(1,97) \\
181(88,72)\end{array}$ & - & $\begin{array}{l}1(0,01) \\
1(0,01) \\
0(0) \\
137(99,98)\end{array}$ & - & $0,0028^{* * *}$ \\
\hline \multicolumn{6}{|l|}{ Manejo profilático } \\
\hline $\begin{array}{l}\text { Compressa com chá de } \\
\text { camomila } \\
\text { Creme de Aloe vera } \\
\text { Outros } \\
\text { Sem registro }\end{array}$ & $\begin{array}{l}63(30,88) \\
0(0) \\
3(1,48) \\
138(67,64)\end{array}$ & - & $\begin{array}{l}1(0,01) \\
117(84,17) \\
7(5,75) \\
14(10,07)\end{array}$ & $0,0017^{*}$ & $0,0001^{* \star *}$ \\
\hline \multicolumn{6}{|l|}{ Tratamento farmacológico } \\
\hline $\begin{array}{l}\text { Esteróide + antibiótico } \\
\text { Esteróide + antibiótico } \\
+ \text { antifúngico } \\
\text { Outros/nenhum/sem registro }\end{array}$ & $\begin{array}{l}57(27,94) \\
10(4,91) \\
137(67,15)\end{array}$ & - & $\begin{array}{l}44(31,65) \\
33(23,75) \\
62(44,60)\end{array}$ & - & $0,0001^{* *}$ \\
\hline
\end{tabular}

${ }^{*}$ Teste exato de Fisher; ${ }^{\star \star}$ Teste do Qui quadrado 


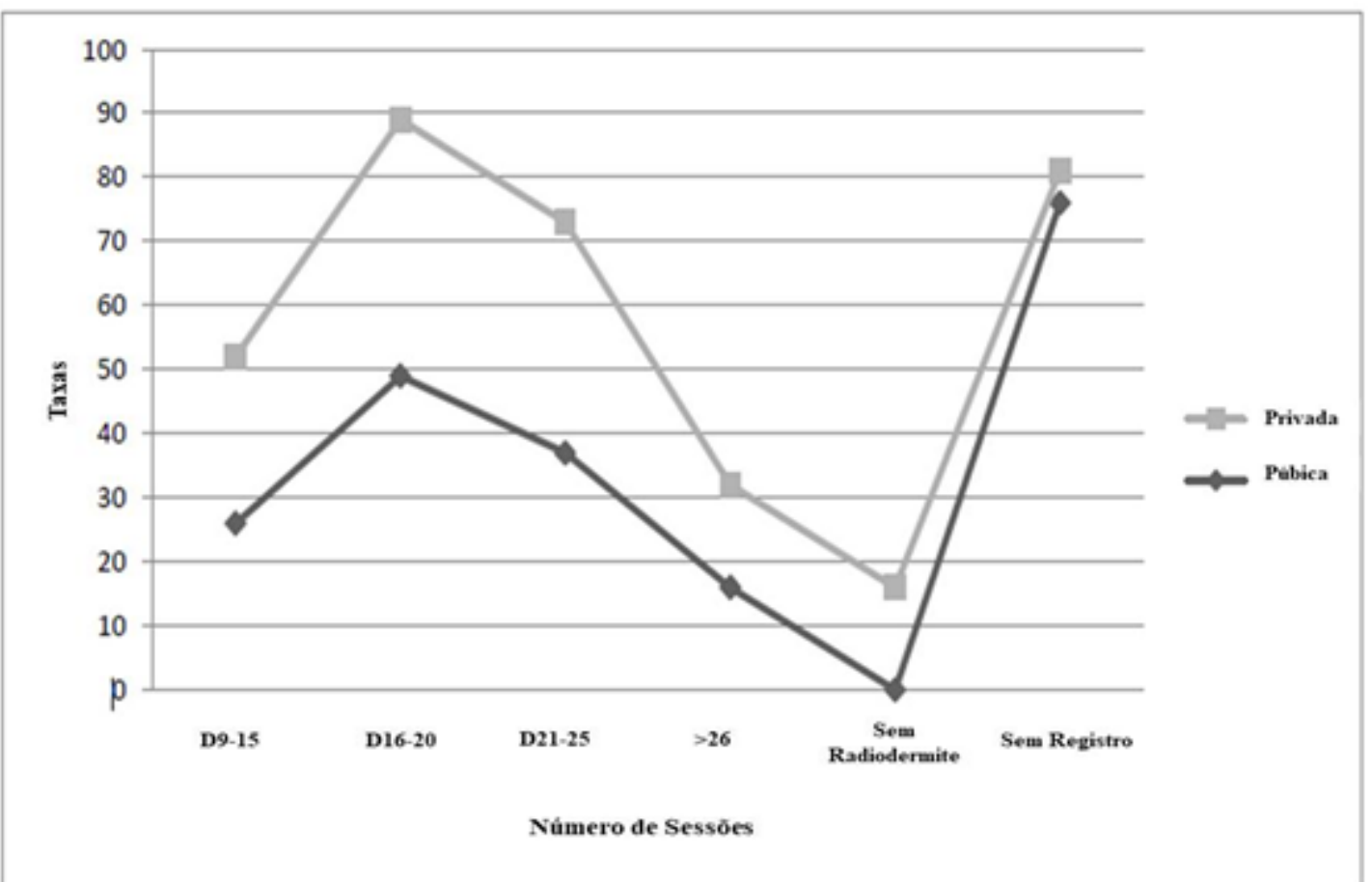

Figura 2 - O número de sessões de radiodermatite nas instituições públicas e privadas. Belém/PA, Brasil, 2012-2015.

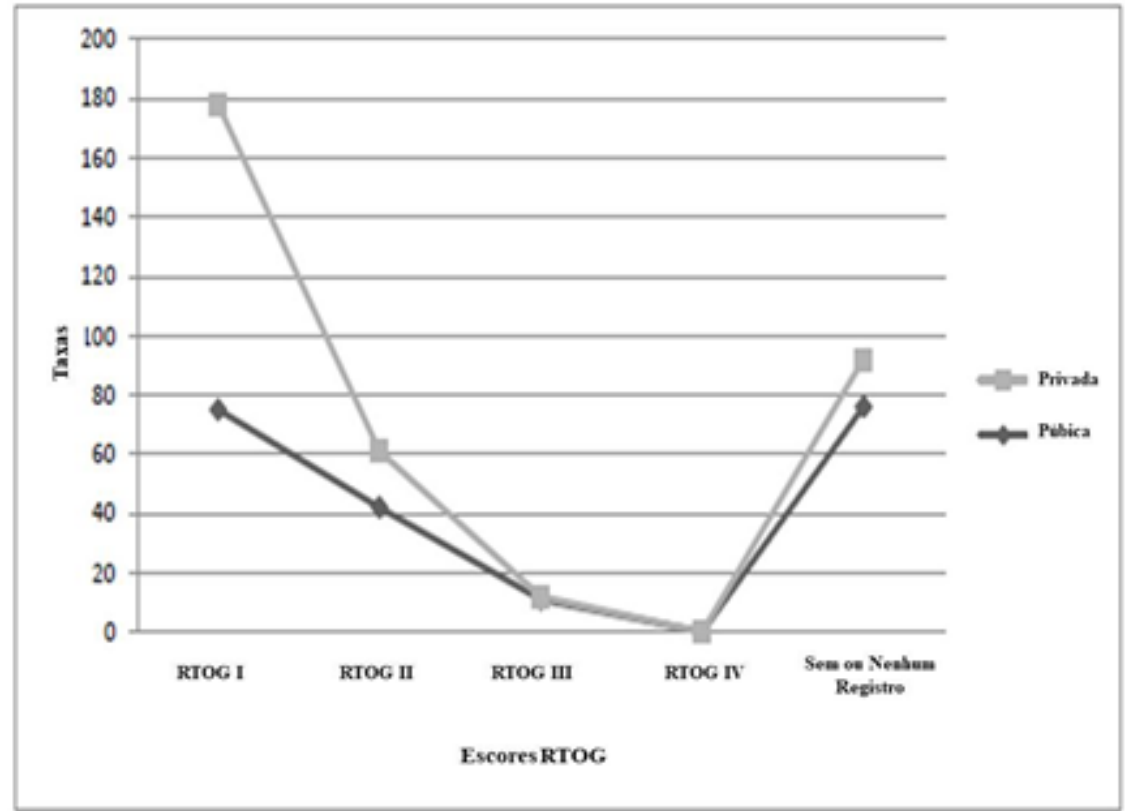

Figura 3 - Grau de RTOG entre casos de radiodermatite aguda em instituições públicas e privadas. Belém/PA, Brasil, 2012-2015.

Os achados revelaram episódios de interrupção temporária do tratamento significativamente maiores na instituição pública em relação a privada $(p=0,0028)$, por 1 semana $(4,9 \%)$ ou 2 semanas $(4,41 \%)$. Os registros nos prontuários exibiram que 67 pacientes $(32,8 \%)$ e $77(55,3 \%)$ apresentaram diferentes graus de descamação úmida inframamária e / ou aréola mamilar em instituições privadas (IMRT) e públicas (3D-CRT), respectivamente (Figura 4). 


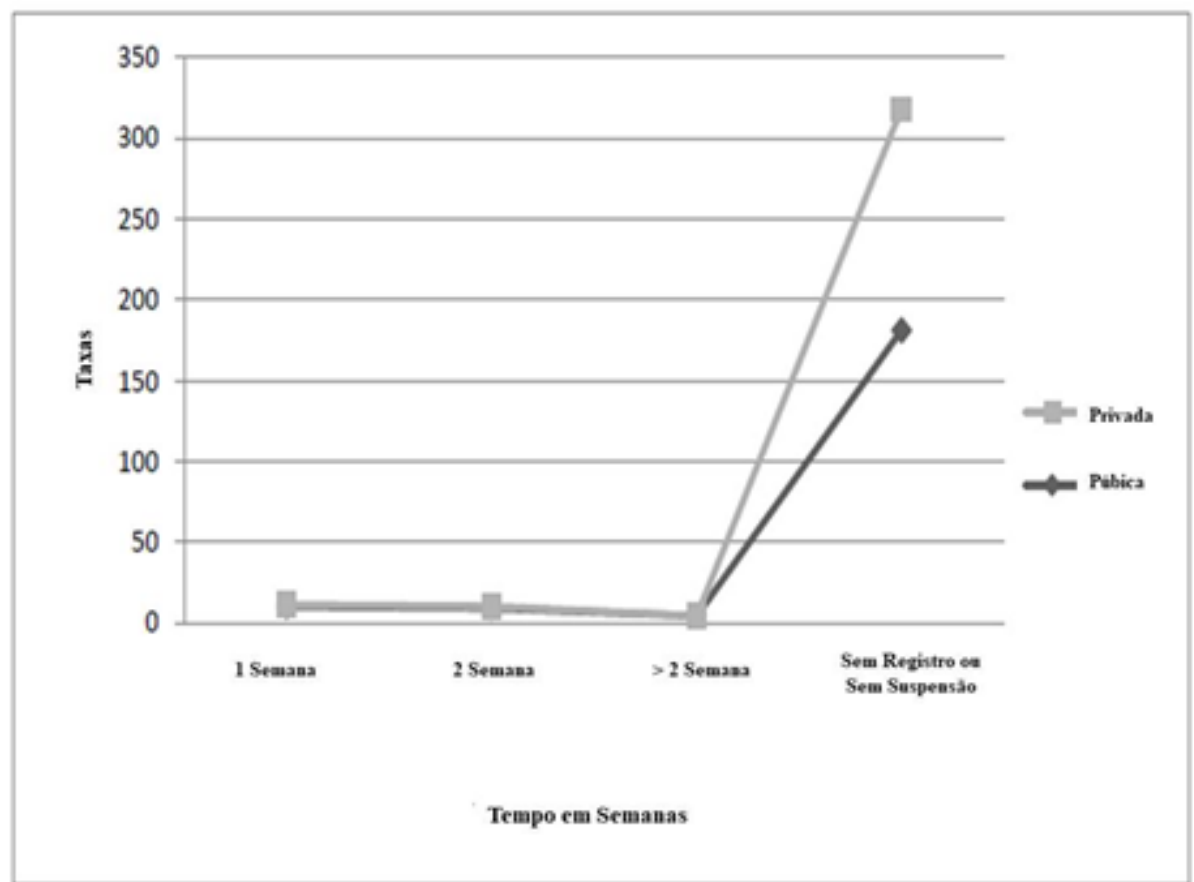

Figura 4 - Interrupção de tratamento secundário à gravidade da radiodermatite aguda em instituições públicas e privadas. Belém/PA, Brasil, 2012-2015.

As instituições divergiram quanto ao manejo profilático $(p=0,0001)$, o chá de camomila (Matricaria chamomilla) foi o mais recomendado pela enfermeira na instituição pública e o creme a base de aloe vera (Aloe vera L.) na privada. Adicionalmente, a maioria do manejo farmacológico coincidiu nas duas instituições, com a prescrição médica de esteróide e antibiótico tópico $(p=0,0001)$.

\section{Discussão}

A radiodermatite aguda é comumente experimentada em mulheres com câncer de mama que recebem radioterapia [5]. O seu surgimento é multifatorial, relacionado a fatores intrínsecos e extrínsecos (principalmente, relacionado a dose total, diária, boost, tipo de energia etc.) [6].

Neste estudo, a caracterização das mulheres foi similar a estudos prévios [10,11]. Os achados deste estudo revelaram informações interessantes. Em primeiro lugar, houve radiodermatite aguda nas duas instituições, independentemente do tipo de tecnologia aplicada, com o pico coincidente entre D16-20 de fração.

Isso pode ser justificado devido a pele estar no trajeto entre a radiação ionizante gerada pelo acelerador e o órgão alvo [10]. A toxicidade cutânea é um evento determinístico, ou seja, é dose-dependente com tempo previsível entre 10-14 dias após o início do tratamento, independente da tecnologia aplicada [8]. Logo, período similar ao nosso.

Em segundo lugar, uma análise mais minuciosa dos casos de radiodermatites na instituição privada mostrou o emprego de uma dose total e fração diária mais elevadas em menor tempo de tratamento (hipofracinamento), o que resultou em graus mais leves de radiodermatite. A distribuição homogênea de dose ao volume alvo pela tecnologia IMRT e entregue de forma hipofracionada é importante na redução da incidência e grau de severidade da radiodermatite [12], dados semelhantes a este estudo.

Por outro lado, a instituição pública aplicou menor dose total e fração diária mais flutuante em mais dias de tratamento (hiperfracionada). Por conseguinte, evidenciou-se radiodermites com diversos graus de severidade, maior número de descamação úmida e, por conseguinte, maior interrupção da radioterapia, cuja repercussão pode ser negativa sobre o prognóstico [2].

A distribuição não homogênea e hiperfracionada são um dos preditores de toxicidade cutânea aguda. Como visto, a tecnologia 3D-CRT $[13,14]$ reforça que a frequência e gravidade da radiodermatite no Sistema Único de Saúde (SUS) está relacionada à técnica terapêutica ultrapassada, ainda guiada por radiografia ou tomografia e os campos conformados ao tumor. Sabe-se ainda que a descamação úmida não ocorre até que uma certa dose limite seja atingida 
e esse risco sofre incremento conforme o volume da pele onde excede esse limiar [10], porém esta variável não foi avaliada neste estudo devido a dados incompletos no prontuário.

Em terceiro lugar, foi observada divergência quanto a recomendação das medidas profiláticas a compressa com chá de camomila (Matricaria recutita Linnaeus) na instituição pública e aloe vera (Aloe vera L.) na privada. Adicionalmente, a maioria do manejo farmacológico coincidiu nas duas instituições, com a prescrição médica de esteróide e antibiótico tópico.

A indicação da camomila foi predominante na primeira instituição devido a apresentar suas propriedades antiinflamatórias, antioxidantes, bactericidas que promove o conforto do paciente, além do fácil acesso e baixo custo desse fitoterápico aos pacientes [15,16]. Já o Aloe vera é uma das plantas mais pesquisadas em cuidados com a pele, sendo eficaz na cicatrização de lesões (inflamação, eritema) e proteção contra radiação UV [17].

Embora a radiodermatite seja um evento comum, não há protocolos clínicos padronizados à prevenção e tratamento. Uma vez que as evidências científicas disponíveis são heterogêneas e não conclusivas, com ensaios clínicos isolados, amostras limitadas, com fins comerciais e lucrativos geralmente [5,9].

Finalmente, o presente estudo apresentou limitações devido ao seu desenho retrospectivo, relacionado ao número relativamente pequeno de pacientes analisados e aos dados incompletos de algumas variáveis (por exemplo, tipo de pele, tamanho da mama e etilismo etc.), bem como a ausência de outras informações relevantes no prontuário, um documento legal. É importante ressaltar que a incidência de radiodermatite é flutuante devido às subnotificações e estudos adicionais que precisam ser desenvolvidos sobre a temática.

Conclusão

Este foi o primeiro relato sobre a experiência institucional quanto à incidência de radiodermatite entre tecnologias diferentes no tratamento do câncer de mama no Norte do Brasil.

$\mathrm{A}$ análise dos prontuários de mulheres submetidas à radioterapia revelou discrepâncias significativas entre as duas instituições, diante de terapia aplicadas e graus de radiodermites, bem como seu manejo. $\mathrm{Na}$ instituição privada, observou-se maior acesso à imunohistoquímica $\mathrm{e}$ maior qualidade assistencial prestada pela enfermeira devido a menor demanda de pacientes. Por outro lado, no hospital público houve maior severidade da lesão e mais interrupção/atraso no tratamento, além de dados incompletos nos prontuários que trazem prejuízo significativo as vertentes clínicas, jurídicas e científicas.

No entanto, a radioterapia de intensidade modulada persiste como um tipo de tecnologia efetiva, porém de alto custo, com acesso restrito a poucos centros no Brasil pelo SUS, ainda ausente nessa região.

1. Ferlay J, Colombet M, Soerjomataram I, Mathers $\mathrm{C}$, Parkin DM, Piñeros $\mathrm{M}$ et al. Estimating the Global Cancer Incidence and Mortality in 2018: Globocan Sources and Methods. Int J Cancer 2019;(8):1941-53. https://doi.org/10.1002/ijc.31937

2. Wei J, Meng L, Hou X, Qu C, Wang B, Xin Y et al. Radiation-induced skin reactions: mechanism and treatment. Cancer Manag Res 2019;(11):167-77. https://doi.org/ 10.2147/CMAR.S188655

3. Bray FN, Simmons BJ, Wolfson AH, Nouri K. Acute and chronic cutaneous reactions to ionizing radiation therapy. Dermatol Ther (Heidelb) 2016;(2):185-206. https://doi.org/10.1007/s13555-016-0120-y

4. Fuzissaki MA, Paiva CE, Oliveira MA, Canto PPL, Maia YCP. The impact of radiodermatitis on breast cancer patients' quality of life during radiotherapy: a prospective cohort study. J Pain Symptom Manage 2019;(58):92-9. https://doi.org/10.1016/j.jpainsymman.2019.03.017

5. Haruna F, Lipsett A, Marignol L. Topical management of acute radiation dermatitis in breast cancer patients: a systematic review and meta-analysis. Anticancer Res 2017;(10):5343-53. https://doi.org/10.21873/anticanres.11960

6. Kitajima M, Mikami K, Noto Y, Itaki C, Fukushi Y, Hirota Y et al. Quantitative assessment of radiodermatitis through a non-invasive objective procedure in patients with breast cancer. Mol Clin Oncol 2020;(12):89-93.

https://doi.org/10.3892/mco.2019.1948 
7. Lee T, Sung KC, Chao PJ, Huang YJ, Lan JH, Wu HY. Relationships among patient characteristics, irradiation treatment planning parameters, and treatment toxicity of acute radiation dermatitis after breast hybrid intensity modulation radiation therapy. PloS One 2018;(13):1-14. https://doi.org/10.1371/journal.pone.0200192

8. Kole A, Kole L, Moran M. Acute radiation dermatitis in breast cancer patients: challenges and solutions. Breast Cancer - Targets and Therapy 2017;(9):313-23. https://doi.org/10.2147/BCTT.S109763

9. Seité S, Bensadoun R, Mazer J. Prevention and treatment of acute and chronic radiodermatitis. Breast Cancer: Targets and Therapy 2017;(9):551-7.

10. Borm KJ, Loos M, Oechsner M, Mayinger MC, Paepke D, Kiechle MB et al. Acute radiodermatitis in modern adjuvant $3 \mathrm{D}$ conformal radiotherapy for breast cancer - the impact of dose distribution and patient related factors. Radiat Oncol 2018;(13):218. https://doi.org/10.1186\%2Fs13014-018-1160-5

11. Mishra R, Khurana R, Mishra H, Rastogi M, Hadi R. Retrospective analysis of efficacy and toxicity of hypo-fractionated radiotherapy in breast carcinoma. J Clin Diagn Res 2016;(10):01-03. https://doi.org/10.7860/JCDR/2016/20769.8350

12. Yee C, Asthana R, Leung E, De Angelis C, Chow E. Radiation-induced skin toxicity in breast cancer patients: a systematic review of randomized trials. Clin Breast Cancer 2018;(5):825-40. https://doi.org/10.1016/i.clbc.2018.06.015

13. Tortorelli G, Di Murro L, Barbarino $R$ et al. Standard or hypofractionated radiotherapy in the postoperative treatment of breast cancer: a retrospective analysis of acute skin toxicity and dose inhomogeneities. BMC Cancer 2013;(13):230. https://doi.org/10.1186/1471-2407-13-230

14. Partl R, Lehner J, Winkler P, Kapp KS. Testing the feasibility of augmented digital skin imaging to objectively compare the efficacy of topical treatments for radiodermatitis. PloS One 2019;(14). https://doi.org/10.1371/journal.pone.0218018

15. Gomes V, Nonato R, Gomes S, Joaquim M, Lago E, Nicolau R. Effects of Matricaria Recutita (L.) in the treatment of oral mucositis. Scientific World Journal 2018;(12):1-8. https://doi.org/10.1155/2018/4392184

16. Miraj S, Alesaeidi S. A systematic review study of therapeutic effects of Matricaria recuitta chamomile (chamomile). Electronic Physician 2016;(8):3024-31. http://doi.org/10.19082/3024

17. Rao S, Hegde S, Beliga-Rao M, Palatty P, George T, Baliga M. An aloe vera-based cosmeceutical cream delays and mitigates ionizing radiation-induced dermatitis in head and neck cancer patients undergoing curative radiotherapy: a clinical study. Medicines (Basel) 2017;(3):44. https://doi.org/10.3390/medicines4030044

18. Union for International Cancer Control. In: Brierley JD, Gospodarowicz MK, Wittekind C eds. The TNM classification of malignant tumours. 8th ed. Wiley Clackwell:2017. $272 \mathrm{p}$.

19. Brasil. Ministério da Saúde, Portaria Conjunta no 04, DE 23 de janeiro de 2018. [Citado em 2019 mar 03]. Disponivel em: https://portalarquivos2.saude.gov.br/images/pdf/2018/fevereiro/07/PORTARIA-no-04PCDT.carcinoma.mama.2018.pdf 